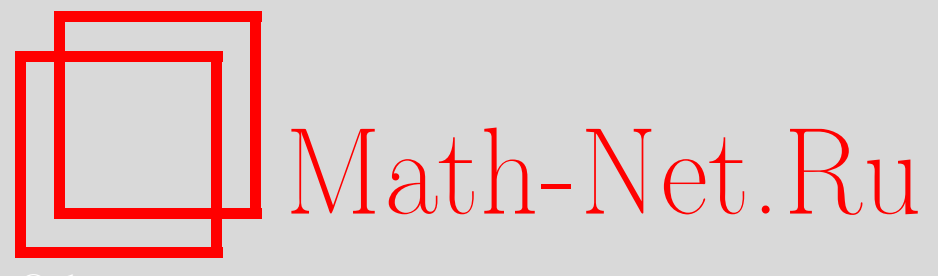

И. Н. Ланджев, Т. Хонольд, Дуги в проективных ельмслевовых плоскостях, Дискрет. матем., 2001, том 13, выпуск 1, 90-109

DOI: https://doi.org/10.4213/dm272

Использование Общероссийского математического портала Math-Net.Ru подразумевает, что вы прочитали и согласны с пользовательским соглашением http://www .mathnet.ru/rus/agreement

Параметры загрузки:

IP : 54.147 .182 .235

26 апреля 2023 г., $12: 58: 41$

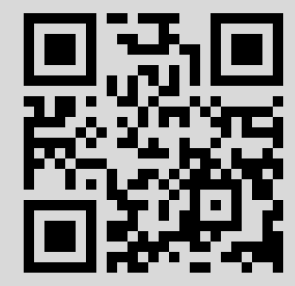


УДК 519.1

\title{
Дуги в проективных ельмслевовых плоскостях
}

\author{
(c) 2001 г. И. Ланджев, Т. Хонольд
}

\begin{abstract}
Изучаются $(k, n)$-дуги в проективных ельмслевовых плоскостях $\operatorname{PHG}\left(R_{R}^{3}\right)$, где $R$ - конечное цепное кольцо. Выводится общая верхняя оценка мощности $k$ такой дуги и указывается максимум мощностей проективных $(k, n)$-дуг для $q^{2} \leqslant n \leqslant q^{2}+q-1$. Приводятся также конструкции проективных дуг в ельмслевовых плоскостях над цепными кольцами порядков 4 и 9.
\end{abstract}

\section{1. Введение}

В последние годы получены существенные продвижения в изучении линейных кодов над конечными кольцами. Ранее, за исключением небольшого числа авторов, интересовались в основном кодами над $Z_{4}$, но теперь увеличивается число работ, связанных с изучением линейных кодов над другими конечными кольцами и модулями (см., например, $[1,2,3,4,5,6,7,8]$, и, в частности, обзор [9]).

В $[10,11]$ мы развивали теорию линейных кодов над конечными цепными кольцами. Это направление можно рассматривать как одно из естественных обобщений теории линейных кодов над конечными полями. В коммутативном случае это часть более общей и также весьма естественной теории линейных кодов над квазифробениусовыми модулями (см. $[1,2,3,9])$

Ассоциативное кольцо с единицей $1 \neq 0$ называется цепным слева (справа), если решетка его левых (правых) идеалов есть цепь. Структура и основные свойства цепных колец описаны в $[12,13,14]$. В частности, в [12] показано, что конечное цепное слева кольцо $R$ является также цепным справа кольцом главных идеалов, каждый его идеал -- двусторонний и является степенью радикала $\operatorname{rad} R$ этого кольца, а также описано строение такого кольца.

Линейные коды над конечными цепными кольцами обладают многими хорошими свойствами, известными для линейных кодов над конечными полями. Кроме того, имеются результаты, показывающие, что, используя хорошие (в некотором смысле) линейные коды над цепными кольцами, можно строить и другие (не обязательно линейные) коды над конечными полями $[15,16,4,7,10]$. В частности, некоторые линейные коды над $Z_{4}$ порождают двоичные нелинейные коды, например, коды Кердока, которые лучше любых двоичных линейных кодов $[15,16]$. В $[17,4,6]$ построен линейный код над кольцом Галуа характеристики 4, из которого подходящей конкатенацией строится обобщенный код Кердока над произвольным полем Галуа характеристики 2. 
В [11] показано, что существует взаимно однозначное соответствие между классами эквивалентных мультимножеств точек в некоторой проективной ельмслевовой геометрии $\mathrm{PHG}\left(R_{R}^{k}\right)$ и классами полулинейно изоморфных так называемых полных линейных кодов, то есть кодов, у которых нет координатных позиций, содержащих элементы только из $\operatorname{rad} R$. C каждым мультимножеством точек из $\operatorname{PHG}\left(R_{R}^{k}\right)$ ассоциируется (левый) линейный код над $R$ с порождающей матрицей, столбцы которой суть столбцы однородных координат точек, составляющих это мультимножество. Наоборот, каждый полный (левый) линейный код над конечным цепным кольцом $R$ позволяет восстановить такое мультимножество точек в $\operatorname{PHG}\left(R_{R}^{k}\right)$. На множестве мультимножеств вводится естественное отношение эквивалентности так, что эквивалентность мультимножеств равносильна полулинейному изоморфизму соответствующих линейных кодов. В связи с этим можно утверждать, что построение в проективной ельмслевовой геометрии мультимножеств максимально возможной мощности, содержащих не более чем заданное число точек из каждой гиперплоскости, позволяет строить линейные коды с интересными свойствами над цепным кольцом.

Однако проблема построения оптимального мультимножества в конечной геометрии интересна сама по себе, она гораздо старше приведенной интерпретации в терминах теории кодов. Имеется обширная литература о множествах точек в классических проективных геометриях над конечными полями $[18,19]$, но результатов о таких множествах в конечных ельмслевовых геометриях авторы не знают.

В этой работе изучаются дуги в проективных ельмслевовых плоскостях $\operatorname{PHG}\left(R_{R}^{3}\right)$, где $R$ - цепное кольцо, $|R|=q^{2}$ и $R / \operatorname{rad} R \cong F_{q}$. Мы ограничиваемся лишь правыми ельмслевовыми плоскостями $\operatorname{PHG}\left(R_{R}^{3}\right)$. Это не нарушает общности, поскольку любой левый $R$-модуль есть правый модуль над двойственным кольцом $R^{\text {орр }}$, которое также есть цепное кольцо из $q^{2}$ элементов с полем вычетов моцности $q$.

\section{2. Основные факты о проективных ельмслевовых плоскостях}

Напомним, что инцидентностной структурой называется тройка $(\mathscr{P}, \mathscr{L}, I)$, состоящая из множества точек $\mathscr{P}$, множества прямых (линий) $\mathscr{L}$ и отношения инцидентности $I \subseteq \mathscr{P} \times \mathscr{L}$. При этом если $(X, s) \in I$, то говорят, что точка $X \in \mathscr{P}$ принадлежит (инцидентна) прямой $s \in \mathscr{L}$, или что прямая $s$ проходит через (содержит) точку $X$, и пишут $X I s$. В качестве примера приведем используемую далее аффинную геометрию $\mathrm{AG}(2, q)$ над полем $F_{q}$, в которой точками являются элементы пространства $F_{q}^{2}$, а прямыми - все одномерные многообразия этого пространства.

Инцидентностная структура называется проективной плоскостью, если она удовлетворяет следующим условиям:

(P1) через любые две точки из $\mathscr{P}$ проходит (единственная) прямая из $\mathscr{L}$;

(P2) любые две прямые из $\mathscr{L}$ пересекаются в единственной точке из $\mathscr{P} ;$

(P3) множество $\mathscr{P}$ содержит четыре точки, находящиеся в общем положении, то есть такие, что никакие три из них не принадлежат одной прямой из $\mathscr{L}$.

Простейший пример проективной плоскости - проективная геометрия $\mathrm{PG}\left(F_{q}^{3}\right)$ над полем $F_{q}$, в которой точки и прямые суть, соответственно, одномерные и двумерные подпространства пространства $F_{q}^{3}$. Далее она обозначается также $\mathrm{PG}(2, q)$. 
В этой геометрии $|\mathscr{P}|=|\mathscr{L}|=q^{2}+q+1$, каждая прямая содержит $q+1$ точек и через каждую точку проходит $q+1$ прямых.

С каждой инцидентностной структурой связано отношение соседства $\propto$, которое задается на множествах $\mathscr{P}, \mathscr{L}$, условиями

(N1) $\forall X, Y \in \mathscr{P}: X \propto Y \Longleftrightarrow \exists s, t \in \mathscr{L}, s \neq t, X I s, X I t, Y I s, Y I t$

(N2) $\forall s, t \in \mathscr{L}: s \propto t \Longleftrightarrow|s \cap t| \geqslant 2$.

Ясно, что для проективной плоскости $\propto$ есть просто отношение равенства.

Проективная ельмслевова плоскость определяется как инцидентностная структура $(\mathscr{P}, \mathscr{L}, I)$, удовлетворяющая следующей системе аксиом:

(PH1) $\forall X, Y \in \mathscr{P}, \exists s \in \mathscr{L}: X I s, Y I s$.

(PH2) $\forall s, t \in \mathscr{L}, \exists X \in \mathscr{P}: X I s, X I t$

(РН3) Отношения $\propto$ на множествах $\mathscr{P}$ и $\mathscr{L}$ суть отношения эквивалентности и для отношения инцидентности $J$, определяемого на элементах $[X],[s]$ фактормножеств, соответственно, $\mathscr{P}^{(1)}=\mathscr{P} / \propto, \mathscr{L}^{(1)}=\mathscr{L} / \propto$, соотношением

$$
[X] J[s] \Longleftrightarrow \exists Q \in[X], t \in[s]: Q I t,
$$

структура $\left(\mathscr{P}(1), \mathscr{L}^{(1)}, J\right)$ есть проективная плоскость.

В условиях последней аксиомы можно рассмотреть каноническое отображение

$$
\pi: \mathscr{P} \rightarrow \mathscr{P}^{(1)}
$$

которое очевидным образом расширяется до отображения

$$
\pi: \mathscr{L} \rightarrow \mathscr{L}^{(1)}
$$

и мы можем говорить, что существует эпиморфизм $\pi$ рассматриваемой инцидентностной структуры на проективную плоскость $\left(\mathscr{P}(1), \mathscr{L}^{(1)}, J\right)$ :

$$
\pi:\left\{\begin{array}{lll}
\mathscr{P} \cup \mathscr{L} & \rightarrow & \mathscr{P}^{(1)} \cup \mathscr{L}^{(1)}, \\
X & \rightarrow & {[X],} \\
l & \rightarrow & {[l] .}
\end{array}\right.
$$

При этом

$$
\begin{gathered}
\forall X, Y \in \mathscr{P}: X \propto Y \Longleftrightarrow \pi(X)=\pi(Y), \\
\forall s, t \in \mathscr{L}: s \propto t \Longleftrightarrow \pi(s)=\pi(t)
\end{gathered}
$$

(см. также [20, 21, 22, 23]).

В данной работе изучается специальный класс проективных ельмслевовых плоскостей, получающихся из свободных модулей ранга $3: R_{R}^{3}$ (или ${ }_{R} R^{3}$ ), где $R-$ конечное цепное кольцо. Пусть $\mathscr{P}$ - множество всех свободных подмодулей модуля $R_{R}^{3}$ ранга $1, \mathscr{L}-$ множество всех свободных подмодулей того же модуля ранга 2 и отношение инцидентности $I \subset \mathscr{P} \times \mathscr{L}$ определяется как теоретико-множественное включение. Тогда $(\mathscr{P}, \mathscr{L}, I)$ есть проективная ельмслевова плоскость, обозначаемая далее через $\mathrm{PHG}\left(R_{R}^{3}\right)$. 
Так как $R$ - квазифробениусово кольцо [24], то для $R$-модулей сохраняются связанные с понятием двойственности соотношения, известные для конечномерных пространств над полями. В частности, каждую прямую $s \in \mathscr{L}$ можно рассматривать как множество свободных ранга 1 подмодулей $\left(x_{1}, x_{2}, x_{3}\right) R$ модуля $R_{R}^{3}$ (множество точек), удовлетворяющих некоторому линейному уравнению

$$
s_{1} x_{1}+s_{2} x_{2}+s_{3} x_{3}=0
$$

в котором по крайней мере один из коэффициентов $s_{i}$ равен 1. Другими словами, двойственная ельмслевова плоскость $\left(\mathscr{L}, \mathscr{P}, I^{\prime}\right)$ (где $I^{\prime}$ есть отношение, транспонированное к $I)$ изоморфна $\operatorname{PHG}\left({ }_{R} R^{3}\right)$.

Две точки $X=\mathrm{x} R$ и $Y=\mathrm{y} R$ являются соседними в $\operatorname{PHG}\left(R_{R}^{3}\right)$, если и только если $|X \cap Y|>1$, то есть система векторов $\mathbf{x}, \mathbf{y}$ линейно зависима над $R$. Соседние прямые характеризуются аналогично, как соседние точки в двойственном пространстве: прямые $s, t \in \mathscr{L}$ являются соседними в точности, если $|s \cap t|>|R|$, то есть если над $R$ линейно зависимы векторы коэффициентов $\left(s_{1}, s_{2}, s_{3}\right),\left(t_{1}, t_{2}, t_{3}\right)$ уравнений, задающих эти прямые. Более того, если мы положим $\bar{R}=R / \operatorname{rad} R$ и для любого множества $S$ точек из $R^{3}$ обозначим через $\bar{S}$ образ $S$ при естественном эпиморфизме $R \rightarrow \bar{R}$, то для любых точек $X, Y \in \mathscr{P}$ и прямых $s, t \in \mathscr{L}$ очевидно, что $\bar{X}$ и $\bar{s}$ суть соответственно одномерное и двумерное подпространства пространства $\bar{R}^{3}$ над полем $\bar{R}=F_{q}$, и как нетрудно проверить, справедливы соотношения

$$
X \propto Y \Longleftrightarrow \bar{X}=\bar{Y}, \quad s \propto t \Longleftrightarrow \bar{s}=\bar{t} .
$$

Таким образом, в рассматриваемой ситуации проективная плоскость $\left(\mathscr{P}^{(1)}, \mathscr{L}^{(1)}, J\right)$ из (РН3) есть не что иное как проективная геометрия

$$
\operatorname{PG}\left(\bar{R}_{\bar{R}}^{3}\right)=(\overline{\mathscr{P}}, \overline{\mathscr{L}}, \bar{I})
$$

над полем $\bar{R}=F_{q}$. Здесь $\overline{\mathscr{P}}, \overline{\mathscr{L}}$ суть соответственно множества одномерных и двумерных подпространств пространства $\bar{R}_{\bar{R}}^{3}$, а $\bar{I}$ - отношение включения.

Как уже отмечалось, мы ограничиваемся случаем, когда $R$ - цепное кольцо из $q^{2}$ элементов с полем вычетов мощности $q$. Это в некотором смысле - основополагающая ситуация, так как для любого цепного кольца $R$ мощности $q^{m}, m>2$, с полем вычетов $F_{q}$ результаты о мультимножествах в проективной ельмслевовой плоскости $\mathrm{PHG}\left(R_{R}^{3}\right)$ базируются на результатах о мультимножествах в классической проективной плоскости $\mathrm{PG}(2, q)$ и в ельмслевовой проективной плоскости над цепным кольцом $R /(\operatorname{rad} R)^{2}$.

Если $q=p^{r}$, то существует ровно $r+1$ классов изоморфных колец с указанными свойствами, один из них представляется кольцом Галуа $\operatorname{GR}\left(q^{2}, p^{2}\right)$ характеристики $p^{2}$, а остальные - факторкольцами колец косых многочленов

$$
F_{q}[X ; \sigma] /\left(X^{2}\right), \quad \sigma \in \operatorname{Aut}\left(F_{q}\right),
$$

каждое из которых имеет характеристику $p$ (см. $[25,12])$. Линейные коды длины $n$ над такими кольцами хорошо отображаются в коды длины $q n$ в $q$-ичном алфавите, как описано в $[4,8,10]$. Если $R$ - одно из колец $F_{q}[X ; \sigma] /\left(X^{2}\right)$, результирующий код также может быть выбран линейным.

Приведем некоторые факты о строении проективных ельмслевовых плоскостей. Следующие результаты в более общей форме содержатся в $[26,20,27,28]$. 
Теорема 1. Пусть $\Pi=(\mathscr{P}, \mathscr{L}, I)=\operatorname{PHG}\left(R_{R}^{3}\right)$, где $R-$ цепное колъцо, $|R|=q^{2} u$ $R / \operatorname{rad} R \cong F_{q}$. Tогдa

(a) $|\mathscr{P}|=|\mathscr{L}|=q^{2}\left(q^{2}+q+1\right)$;

(b) каждая точка (прямая) имеет $q^{2}$ соседних точек (прямых);

(c) каждая точка (прямая) инцидентна точно $q(q+1)$ прямым (точкам);

(d) для заданных инцидентных точки $P$ и прямой $l$ существует точно q точек на $l$, являющихся соседними для $P$, и точно $q$ прямых, проходящих через точку $P$, являющихся соседними для $l$.

Доказательство. Докажем (а). Каждая точка представима в точности в одной из следующих трех форм:

$$
\begin{aligned}
& (1, a, b) R, \quad a, b \in R \\
& (a, 1, b) R, \quad a \in \operatorname{rad} R, \quad b \in R \\
& (a, b, 1) R, \quad a, b \in \operatorname{rad} R .
\end{aligned}
$$

Теперь результат о числе точек получается простым подсчетом. Число прямых подсчитывается аналогично, путем их представления уравнениями с указанными наборами коэффициентов.

Докажем (b). Пусть $P=(1,0,0) R$. Тогда все соседние к $P$ точки суть все точки вида $(1, a, b) R$, где $a, b \in \operatorname{rad} R$. Остальные случаи рассматриваются аналогично.

Докажем (c). Допустим опять, что $P=(1,0,0) R$. Тогда все различные прямые, проходящие через $P$, суть все прямые, которые задаются уравнениями

$$
\begin{array}{ll}
x_{2}+a x_{3}=0, & a \in R, \\
b x_{2}+x_{3}=0, & b \in \operatorname{rad} R .
\end{array}
$$

Наконец, докажем (d). Если, например, $P=(1,0,0) R$ и прямая $l$ задается уравнением $x_{3}=0$, то соседние с $P$ точки на $l$ суть все точки вида $(1, a, 0) R$, где $a \in \operatorname{rad} R$.

Зафиксируем точку $P \in \mathscr{P}$ и обозначим через $\mathscr{L}([P])$ множество прямых из $\mathscr{L}$, каждая из которых содержит по крайней мере одну точку, а следовательно, ввиду теоремы $1(\mathrm{~d})$, ровно $q$ точек из $[P]$. Для двух линий $s, t \in \mathscr{L}([P])$ будем писать $s \sim t$, если $s$ и $t$ совпадают на множестве $[P]$. Зафиксируем и обозначим через $\mathscr{L}^{\prime}$ множество линий, содержащее по одному представителю из каждого класса эквивалентности относительно $\sim$.

Следующая теорема из [27] (см. теорему 1(a)) есть часть гораздо более общего результата. Ниже мы отождествляем, для краткости, точки $X \in \mathscr{P}$ с векторами, порождающими соответствующий подмодуль, и вместо $X=\mathbf{x} R$ пишем $X=\mathbf{x}$

Теорема 2. Инцидентностная структура $\left([P], \mathscr{L}^{\prime},\left.I\right|_{\left.[P] \times \mathscr{L}^{\prime}\right)}\right.$ изоморфна аффинной плоскости $\mathrm{AG}(2, q)$ над $F_{q}$.

Доказательство. Пусть $P=(1,0,0)$. Тогда $[P]$ есть множество точек $Q$ вида

$$
Q=(1, a \theta, b \theta), \quad a, b, \in \Gamma,
$$


где $\Gamma$ - некоторая фиксированная полная система представителей смежных классов по модулю $\operatorname{rad} R$ и $\operatorname{rad} R=R \theta=\theta R$. Элементы множества $\Gamma$ можно рассматривать как элементы поля $F_{q}$. Тогда изоморфизм $\varphi$ рассматриваемой структуры на соответствующую аффинную плоскость задается равенством

$$
\varphi((1, a \theta, b \theta))=(a, b) .
$$

Для $X \in \mathscr{P}, l \in \mathscr{L}$ условимся писать $X \propto l$, если существует точка $Y \in \mathscr{P}$ такая, что $Y I l$ и $X \propto Y$. Ясно, что для $\Pi=\operatorname{PHG}\left(R_{R}^{3}\right)$ верны соотношения

$$
X \propto l \Longleftrightarrow \bar{X} \bar{I} l \Longleftrightarrow \bar{X} \subset \bar{l} \text {. }
$$

Зафиксируем в $\Pi$ прямую $l \in \mathscr{L}$ и определим новое множество точек $\mathfrak{P}$ равенстBOM

$$
\begin{aligned}
\mathfrak{P} & =\{s \cap[X] \mid s \in \mathscr{L}, X \in \mathscr{P}, s \propto l, X \propto l\} \cup\left\{P_{\infty}\right\} \\
& =\{s \cap[X] \mid s \in \mathscr{L}, X \in \mathscr{P}, \bar{s}=\bar{l}, \bar{X} \subset \bar{l}\} \cup\left\{P_{\infty}\right\} .
\end{aligned}
$$

Зададим отношение инцидентности $\mathfrak{I} \subseteq \mathfrak{P} \times \mathscr{L}$ условием

$$
\begin{aligned}
(s \cap[X]) \mathfrak{I} t & \Longleftrightarrow t \cap(s \cap[X]) \neq \varnothing, \\
\left(P_{\infty}\right) \mathfrak{I} t & \Longleftrightarrow t \phi l .
\end{aligned}
$$

Будем называть прямые $l_{1}, l_{2} \in \mathscr{L}$ эквивалентными и писать $l_{1} \sim l_{2}$, если множества точек из $\mathfrak{P}$, инцидентных относительно $\mathfrak{I}$ этим прямым, совпадают. Зафиксируем и обозначим через $\mathfrak{L}$ множество прямых из $\mathscr{L}$, содержащее по одному представителю из каждого класса прямых, эквивалентных относительно . Следующую теорему, в несколько иной форме, можно найти в [27] (см. предложение 1(b)) и в [28] (см. предложение 3.2 ).

Теорема 3. Инцидентностная структура $\left(\mathfrak{P}, \mathfrak{L},\left.\mathfrak{I}\right|_{\mathfrak{P} \times \mathfrak{L})}\right.$ изоморфна проективной геометрии $\mathrm{PG}(2, q)$ над полем $F_{q}$.

Доказательство. Пусть $l$ задается уравнением $x_{3}=0$. Тогда множество точек $P \in$ $\mathscr{P}$, инцидентных какой либо прямой из $[l]$ есть множество точек вида

$$
P=\left(p_{1}, p_{2}, p_{3} \theta\right) R, \quad p_{1}, p_{2} \in R, \quad p_{3} \in \Gamma,
$$

где $Г$ - такая же полная система представителей, как и в теореме 2. Нетрудно проверить, что функция

$$
\varphi: \begin{cases}\left(p_{1}, p_{2}, p_{3} \theta\right) & \rightarrow\left(\bar{p}_{1}, \bar{p}_{2}, \bar{p}_{3}\right) \\ P_{\infty} & \rightarrow(0,0, \overline{1})\end{cases}
$$

есть константа на каждом множестве $s \cap[X]$ точек $P$ (то есть на каждой точке из $\mathfrak{P})$ и она индуцирует искомый изоморфизм рассматриваемой инцидентностной структуры на $\mathrm{PG}(2, q)$.

Пример 1. Здесь мы опишем структуру $\left(\mathfrak{P}, \mathfrak{L},\left.\mathfrak{I}\right|_{\mathfrak{P} \times \mathfrak{L}}\right)$ для случая, когда $R=Z_{4}$ и прямая $l$ задается уравнением $x_{3}=0$. Множество точек $\mathfrak{P}$ состоит из $P_{\infty}$ и точек

$$
\begin{array}{lll}
Q_{1}=\{(1,0,0),(1,2,0)\}, & Q_{2}=\{(1,0,2),(1,2,2)\}, & Q_{3}=\{(0,1,0),(2,1,0)\}, \\
Q_{4}=\{(0,1,2),(2,1,2)\}, & Q_{5}=\{(1,1,0),(1,3,0)\}, & Q_{6}=\{(1,1,2),(1,3,2)\},
\end{array}
$$


множество прямых $\mathfrak{L}$ состоит из следующих подмножеств:

$$
\begin{array}{llll}
\left\{Q_{1}, Q_{3}, Q_{5}\right\}, & \left\{Q_{1}, Q_{4}, Q_{6}\right\}, & \left\{Q_{2}, Q_{3}, Q_{6}\right\}, & \left\{Q_{2}, Q_{4}, Q_{5}\right\} \\
\left\{P_{\infty}, Q_{1}, Q_{2}\right\}, & \left\{P_{\infty}, Q_{3}, Q_{4}\right\}, & \left\{P_{\infty}, Q_{5}, Q_{6}\right\} . &
\end{array}
$$

Нетрудно видеть, что описанная структура изоморфна $\operatorname{PG}(2,2)$ и изоморфизм $\varphi$ из теоремы 3 задается следующим образом:

$$
\begin{aligned}
& \varphi\left(Q_{1}\right)=(1,0,0), \quad \varphi\left(Q_{2}\right)=(1,0,1), \quad \varphi\left(Q_{3}\right)=(0,1,0), \\
& \varphi\left(Q_{4}\right)=(0,1,1), \quad \varphi\left(Q_{5}\right)=(1,1,0), \quad \varphi\left(Q_{6}\right)=(1,1,1), \\
& \varphi\left(P_{\infty}\right)=(0,0,1) .
\end{aligned}
$$

\section{3. Дуги и блочные мультимножества в проективных ельмслевовых плоскостях}

Пусть $\Pi=(\mathscr{P}, \mathscr{L}, I)-$ проективная ельмслевова плоскость.

Определение 1. Мультимножеством в П называется отображение $\mu: \mathscr{P} \rightarrow \mathbf{N}_{0}$.

При этом целое неотрицательное число $\mu(P)$ называется кратностью точки $P$. Мы можем расширить область определения функции $\mu$ на множество подмножеств множества $\mathscr{P}$, полагая

$$
\mu(\mathscr{Q})=\sum_{P \in \mathscr{Q}} \mu(P), \quad \mathscr{Q} \subseteq \mathscr{P}
$$

Число

$$
\mu(\mathscr{P})=\sum_{P \in \mathscr{P}} \mu(P)
$$

называется мощностью (или длиной, или размером) мультимножества $\mu$. Носитель Supp $\mu$ мультимножества $\mu$ определяется как

$$
\operatorname{Supp} \mu=\{P \in \mathscr{P} \mid \mu(P)>0\} \text {. }
$$

Мультимножество $\mu$ следующим естественным образом индуцирует мультимножество $\mu^{(1)}$ на проективной факторплоскости $\pi(\Pi)$ :

$$
\mu^{(1)}:\left\{\begin{array}{lll}
\mathscr{P}^{(1)} & \rightarrow & \mathbf{N}_{0} \\
{[P]} & \rightarrow & \mu([P]) .
\end{array}\right.
$$

Определение 2. Мультимножества $\mu^{\prime}$ и $\mu^{\prime \prime}$ в проективных ельмслевовых плоскостях, соответственно $\Pi^{\prime}=\left(\mathscr{P}^{\prime}, \mathscr{L}^{\prime}, I^{\prime}\right)$ и $\Pi^{\prime \prime}=\left(\mathscr{P}^{\prime \prime}, \mathscr{L}^{\prime \prime}, I^{\prime \prime}\right)$, называются эквивалентными, если существует изоморфизм $\sigma: \Pi^{\prime} \rightarrow \Pi^{\prime \prime}$ такой, что $\mu^{\prime}(P)=\mu^{\prime \prime}(\sigma P)$ для каждого $P \in \mathscr{P}^{\prime}$.

Определение 3. Мультимножество $\mu: \mathscr{P} \rightarrow \mathbf{N}_{0}$ называется $(k, n)$-дугой, если $\mu(\mathscr{P})=k$ и $\mu(l) \leqslant n$ для любой прямой $l \in \mathscr{L}$. Такая дуга называется полной, если не существует $\left(k^{\prime}, n\right)$-дуги $\mu^{\prime}$ такой, что $k^{\prime}>k$ и $\mu^{\prime}(P) \geqslant \mu(P)$ для каждой точки $P \in \mathscr{P}$. 
Определение 4. Мультимножество $\mu: \mathscr{P} \rightarrow \mathbf{N}_{0}$ называется $(t, s)$-блочным мультимножеством, если $\mu(\mathscr{P})=t$ и $\mu(l) \geqslant s$ для любой прямой $l \in \mathscr{L}$. Такое мультимножество называется неприводимым, если не существует $\left(t^{\prime}, s\right)$-блочного мультимножества $\mu^{\prime}$, удовлетворяющего условиям $t^{\prime}<t$ и $\mu^{\prime}(P) \leqslant \mu(P)$ для всех $P \in \mathscr{P}$.

Дуга (блочное мультимножество) $\mu$ со свойством $\mu(P) \in\{0,1\}$ для всех $P \in \mathscr{P}$ называется проективной. Проективные дуги и блочные мультимножества можно рассматривать как множества точек, отождествляя их с их носителями. В этом разделе все дуги и блочные мультимножества, за небольшим исключением (теоремы 4,5 и 6), являются проективными. Для любого натурального $n$ обозначим через $m_{n}\left(R_{R}^{3}\right)$ максимальное натуральное $k$ такое, что в $\operatorname{PHG}\left(R_{R}^{3}\right)$ существует $(k, n)$-дуга.

Теорема 4. Пусть $|R|=q^{2}, \bar{R} \cong F_{q} u \mu: \mathscr{P} \rightarrow \mathrm{N}_{0}$ ecmъ $(k, n)$-дуга в $\mathrm{PHG}\left(R_{R}^{3}\right)$. Допустим, что для некоторого класса соседних точек $[P]$ выполнлетсл равенство $\mu([P])=u$. Тогдa

(a) $k \leqslant q^{2}(n-1)+q(n-u)+u$;

(b) $k \leqslant q(q+1)(n-\lceil u / q\rceil)+u$.

Доказательство. Докажем (a). Пусть $Q \in[P], \mu(Q)>0$. По теореме $1(\mathrm{~d})$ семейство из $q(q+1)$ прямых, проходящих через $Q$, разбивается на $q+1$ непересекающихся классов $\mathscr{L}_{i}(Q), 1 \leqslant i \leqslant q+1$, каждый из которых содержит $q$ прямых, совпадающих на $[P]$. Пусть

$$
b_{i}=\mu([P] \cap l), \quad l \in \mathscr{L}_{i}(Q) .
$$

Подсчет кратностей точек на прямых аффинной плоскости, определенной на $[P]$ в теореме 2 дает неравенство

$$
\sum_{i=1}^{q+1} b_{i}=u+q \mu(Q) \geqslant u+q
$$

Кроме того, справедливо неравенство

$$
\sum_{l \in \mathscr{L}_{i}(Q)} \mu(l \backslash[P]) \leqslant q\left(n-b_{i}\right) .
$$

Отсюда следует, что

$$
\begin{aligned}
k & =\mu([P])+\sum_{i=1}^{q+1} \sum_{l \in \mathscr{L}_{i}(Q)} \mu(l \backslash[P]) \leqslant u+q \sum_{i=1}^{q+1}\left(n-b_{i}\right) \\
& \leqslant u+q(q+1) n-q(u+q)=q^{2}(n-1)+q(n-u)+u .
\end{aligned}
$$

Докажем теперь второе утверждение. Рассмотрим классы $\left[l_{i}\right], i=1, \ldots, q+1$, соседних линий, проходящих через точки из $[P]$. Для каждого $i$ существует линия $l \in\left[l_{i}\right]$ со свойством

$$
\mu(l \cap[P]) \geqslant\lceil u / q\rceil
$$

Следовательно,

$$
\sum_{[Q] \in\left[l_{i}\right] \backslash\{[P]\}} \mu([Q]) \leqslant q(n-\lceil u / q\rceil) .
$$

4 Дискретная математика, т.13 №1 
Отсюда следуют соотношения

$$
\begin{aligned}
k & =\sum_{[X] \in \mathscr{P}^{(1)}} \mu([X])=\mu([P])+\sum_{i=1}^{q+1} \sum_{[Q] \in\left[l_{i}\right] \backslash\{[P]\}} \mu([Q]) \\
& \leqslant u+q(n-\lceil u / q\rceil)(q+1) .
\end{aligned}
$$

Следствие 1. Справедливо неравенство

$m_{n}\left(R_{R}^{3}\right) \leqslant \max _{1 \leqslant u \leqslant q^{2}} \min \left\{u\left(q^{2}+q+1\right), q^{2}(n-1)+q(n-u)+u, q(q+1)(n-\lceil u / q\rceil)+u\right\}$.

Для значений $n$, близких к $q^{2}+q$, можно указать точное значение $m_{n}\left(R_{R}^{3}\right)$. Для этого нам нужна теорема, представляющая собой компиляцию известных результатов о блочных множествах (см., например, $[18,19])$.

Теорема 5. Пусть $\widehat{\Pi}=(\widehat{\mathscr{P}}, \widehat{\mathscr{L}}, \widehat{I})=\mathrm{PG}(2, q), q=p^{m}$, где $m-$ натуралъное число, u nycms

$$
\mu: \widehat{\mathscr{P}} \rightarrow \mathbf{N}_{0}
$$

есть $(t, s)$-блочное мулътимножество, причем $s \leqslant q$. Тогда $t \geqslant s(q+1)$, и если $t=s(q+1), s<p$, то существуют, не обязателъно различные, прямые $l_{1}, \ldots, l_{s}$, maxue, чmo

$$
\mu(P)=\left|\left\{j \in\{1, \ldots, s\} \mid P \widehat{I} l_{j}\right\}\right| .
$$

Доказателъство. Если $t<s(q+1)$, то существует точка $P \in \mathscr{P}$ кратности 0 (0-точка), тогда противоположное неравенство получается путем подсчета кратностей прямых, проходящих через $P$.

Пусть $t=s(q+1)$. В таком случае в $\mathscr{P}$ также существуют 0-точки и все прямые, проходящие через фиксированную 0-точку имеют кратность $s$. Заметим, что при этом условии $\mathscr{P}$ содержит прямую без 0-точек. Действительно, в противном случае каждая прямая в $\mathscr{P}$ имеет кратность $s$, и так как каждая точка $P \in \mathscr{P}$ принадлежит ровно $q+1$ прямым, должно выполняться равенство

$$
\sum_{l \in \mathscr{L}} \mu(l)=(q+1) \sum_{P \in \mathscr{P}} \mu(P),
$$

то есть $s\left(q^{2}+q+1\right)=s q(q+1)$, что невозможно.

Покажем сначала, что при условии $s<p$ кратность любой прямой $l$, не содержащей 0-точек, удовлетворяет неравенству $\mu(l) \geqslant q+s$.

Пусть $l_{0}$ - прямая без 0-точек. Если удалить из $\mathrm{PG}(2, q)$ эту прямую со всеми ее точками, то получится аффинная плоскость $\mathrm{AG}(2, q)$, точки которой мы отождествим с элементами поля $F_{q^{2}}$. Это можно сделать, например, следующим образом. Без потери общности рассуждений можно считать, что $l_{0}$ состоит из точек $\left(x_{1}, x_{2}, x_{3}\right)$, для которых $x_{3}=0$. Тогда все точки в $\mathrm{PG}(2, q) \backslash l_{0}$ имеют вид $\left(x_{1}, x_{2}, 1\right)$ и мы можем установить соответствие между точками $\mathrm{PG}(2, q) \backslash l_{0}$ и элементами $F_{q^{2}}$ правилом

$$
\left(x_{1}, x_{2}, 1\right) \rightarrow x_{1}+x_{2} \theta,
$$

где $\theta$ - произвольный элемент из $F_{q^{2}} \backslash F_{q}$. Кроме того, можно считать, что при этом соответствии нуль поля $F_{q^{2}}$ не является 0-точкой, то есть тройка $(0,0,1)$ не является 0-точкой в $\mathrm{PG}(2, q)$. Это возможно, поскольку группа автоморфизмов (коллинеаций) проективной плоскости транзитивна на четверках точек, находящихся в 
общем положении, и если $(0,0,1)$ есть 0-точка, то можно применить автоморфизм, оставляющий неподвижной прямую $l_{0}$ и переводящий $(0,0,1)$ в выбранную точку ненулевой кратности.

Рассмотрим прямую $l$ в аффинной плоскости $\mathrm{PG}(2, q) \backslash l_{0}$. Пусть $X, Y \in F_{q^{2}}$ две точки принадлежащие $l$. Тогда элемент $(X-Y)^{q-1}$ есть корень степени $q+1$ из 1 , который зависит только от $l$ и не зависит от выбора точек. Действительно, прямая $l=\langle X, Y\rangle$ есть множество точек вида $t X+(1-t) Y$, где $t$ пробегает $F_{q}$. Если $V, W-$ другая пара точек на $l$, то

$$
V=v X+(1-v) Y, \quad W=w X+(1-w) Y, \quad v, w \in F_{q}
$$

и

$$
\begin{aligned}
(V-W)^{q-1} & =((v-w) X-(v-w) Y)^{q-1} \\
& =(v-w)^{q-1}(X-Y)^{q-1}=(X-Y)^{q-1}
\end{aligned}
$$

Таким образом, корректно обозначение

$$
U(l)=(X-Y)^{q-1} .
$$

Пусть $l_{1}, l_{2}$ - две прямые из $\mathrm{PG}(2, q)$, отличные от $l_{0}$, и $l^{\prime}, l^{\prime \prime}$ - их образы в аффинной плоскости $\mathrm{PG}(2, q) \backslash l_{0}$. Покажем, что прямые $l^{\prime}$ и $l^{\prime \prime}$ параллельны, в точности если $U\left(l^{\prime}\right)=U\left(l^{\prime \prime}\right)$. Другими словами, прямые $l_{1}$ и $l_{2}$ пересекаются на прямой $l_{0}$ тогда и только тогда, когда $U\left(l^{\prime}\right)=U\left(l^{\prime \prime}\right)$. Пусть $l^{\prime}$ и $l^{\prime \prime}$ не параллельны. Тогда для подходящих $X, Y, Z \in F_{q^{2}}$

$$
X=l^{\prime} \cap l^{\prime \prime}, \quad l^{\prime}=\langle X, Y\rangle, \quad l^{\prime \prime}=\langle X, Z\rangle .
$$

Если $U\left(l^{\prime}\right)=U\left(l^{\prime \prime}\right)$, то есть

$$
(X-Y)^{q-1}=(X-Z)^{q-1}
$$

To

$$
((X-Y) /(X-Z))^{q-1}=1
$$

Следовательно,

$$
(X-Y) /(X-Z)=t \in F_{q}, \quad Y=(1-t) X+t Z,
$$

и точки $X, Y, Z$ коллинеарны, что противоречит условию $l^{\prime} \neq l^{\prime \prime}$. Обратное утверждение доказывается сходными рассуждениями.

Таким образом, мы имеем естественное отождествление точек прямой $l_{0}$ с элементами группы $\mathscr{U}$ всех корней степени $(q+1)$ из единицы в поле $F_{q^{2}}$. Заданное мультимножество определяет для каждой точки $P \in F_{q^{2}}$, как для точки из $\mathrm{PG}(2, q) \backslash l_{0}$, кратность $\mu(P)$. Кроме того, для точек $U \in \mathscr{U}$, в случае, если они рассматриваются как точки из $l_{0}$, также определена кратность, вообе говоря, отличная от $\mu(U)$, которую мы будем обозначать $\mu_{0}(U)$.

Рассмотрим многочлен

$$
\begin{aligned}
F(x, y) & =\prod_{P \in F_{q^{2}}}\left(1-(1-P x)^{q-1} y\right)^{\mu(P)} \prod_{U \in \mathscr{U}}\left(1-U x^{q-1} y\right)^{\mu_{0}(U)} \\
& =\sum_{i=0}^{s(q+1)} F_{i}(x) y^{i} .
\end{aligned}
$$


Пусть $T \in F_{q^{2}}-$ произвольная 0-точка в $\mathrm{PG}(2, q) \backslash l_{0}$. Тогда

$$
\left(1-P T^{-1}\right)^{q-1}=U T^{1-q}
$$

где $U \in \mathscr{U}$ элемент, зависящий только от прямой $\langle T, P\rangle$. Так как все прямые в $\operatorname{PG}(2, q)$, проходящие через $T$, имеют одну и ту же кратность $s$, получаем, что

$$
\begin{aligned}
F\left(T^{-1}, y\right) & =\prod_{U T^{1-q} \in \mathscr{U}}\left(1-U T^{1-q} y\right)^{s} \\
& =\prod_{U \in \mathscr{U}}(1-U y)^{s}=\left(1-y^{q+1}\right)^{s} .
\end{aligned}
$$

В частности, $F_{1}\left(T^{-1}\right)=0$. Так как $\operatorname{deg} F_{1} \leqslant q-1$, а число 0-точек $T$ есть

$$
q^{2}+q+1-s(q+1) \geqslant q^{2}+q+1-(q-1)(q+1)=q+2,
$$

то $F_{1}(x) \equiv 0$. Теперь из равенства

$$
\begin{aligned}
F(0, y) & =\prod_{P \in F_{q^{2}}}(1-y)^{\mu(P)}=(1-y)^{s(q+1)-\mu\left(l_{0}\right)} \\
& =1-\left(\begin{array}{c}
s(q+1)-\mu\left(l_{0}\right) \\
1
\end{array}\right) y+\left(\begin{array}{c}
s(q+1)-\mu\left(l_{0}\right) \\
2
\end{array}\right) y^{2}-\ldots
\end{aligned}
$$

следует, что

$$
\mu(l) \equiv s(q+1) \equiv s \bmod p .
$$

Отсюда, так как $\mu\left(l_{0}\right) \geqslant q+1, s<p$, следует нужное неравенство

$$
\mu\left(l_{0}\right) \geqslant q+s .
$$

Теперь доказательство второй части теоремы завершается индукцией по $s$. В случае $s=1$ утверждение тривиально: $(q+1,1)$-блочное множество есть множество точек некоторой прямой, а именно, прямой без 0-точек. Допустим, что $\mu$ есть $((s(q+1), s)$-блочное мультимножество и $1<s<p$. Выберем в $\mathscr{P}$ прямую $l$ без 0 -точек и уменьшим на 1 кратность каждой точки на этой прямой. Тогда по доказанному новая кратность прямой $l$ не меньше $s-1$ и мы получаем $((s-1)(q+1), s-1)$ блочное мультимножество $\mu^{\prime}$. По предположению индукции существуют прямые $l_{1}, \ldots, l_{s-1} \in \mathscr{L}$ такие, что

$$
\mu^{\prime}(P)=\left|\left\{j \in\{1, \ldots, s-1\} \mid P \widehat{I} l_{j}\right\}\right| .
$$

Добавление к этим прямым прямой $l_{s}=l$ дает нужную систему прямых для исходного мультимножества.

Теорема 6. Пусть $|R|=q^{2}, \bar{R} \cong F_{q}$, и пусть $\mu$ - проективное $(t, s)$-блочное мультимножество в

$$
\Pi=(\mathscr{P}, \mathscr{L}, I)=\operatorname{PHG}\left(R_{R}^{3}\right), \quad 1 \leqslant s \leqslant q
$$

Tогдa

$$
t \geqslant s q(q+1)
$$


причем равенство возможно для любого $s=1, \ldots, q$. При условии

$$
t=s q(q+1), \quad s<p
$$

в П существуют прямъе, скажем $l_{1}, \ldots, l_{s}$ (не облзателъно различные) такие, чmo

$$
\mu^{(1)}([P])=q\left|\left\{j \mid[P] J\left[l_{j}\right]\right\}\right| .
$$

Доказательство. Прежде всего заметим, что для любого $s=1, \ldots, q$ в рассматриваемой плоскости действительно существует проективное $(s q(q+1), s)$-блочное мультимножество. Рассмотрим прямую $l \in \mathscr{L}$ и соответствующую ей инцидентностную структуру

$$
\left(\mathfrak{P}, \mathfrak{L},\left.\mathfrak{I}\right|_{\mathfrak{P} \times \mathfrak{L}}\right)=\operatorname{PG}(2, q),
$$

огределенную в теореме 3 . Выберем в этой структуре $s(q+1)$ различных точек $\left\{Q_{j}\right\}_{j=1}^{s(q+1)}, Q_{j} \neq P_{\infty}$, так, чтобы выполнялись два условия: среди выбранных точек существует $q+1$ коллинеарных; каждая прямая из $\mathfrak{L}$, проходящая через $P_{\infty}$, содержит ровно $s$ точек $Q_{j}$. Такое множество легко построить, выбрав сначала $q+1$ коллинеарных точек, то есть прямую в $\mathrm{PG}(2, q)$, и добавляя к ним по $s-1$ точек с каждой прямой, проходящей через $P_{\infty}$.

Теперь искомое проективное $(s q(q+1), s)$-блочное мультимножество $\mu$ в П можно определить следующим образом:

$$
\mu(P)= \begin{cases}1, & \text { если } P \in Q_{j} \text { для некоторого } j \in\{1, \ldots, s(q+1)\} \\ 0 & \text { в противном случае. }\end{cases}
$$

Пусть $\mu$ - проективное $(t, s)$-блочное мультимножество в П. Если существует класс соседних точек $[X]$ такой, что

$$
\mu([X])=a, \quad 0<a<q,
$$

то, повторяя рассуждения из доказательства пункта (b) теоремы 4 , можно получить неравенство

$$
\mu(\mathscr{P})=a+s q(q+1)>s q(q+1) .
$$

Если все классы точек удовлетворяют условию $\mu([X])>0$, то

$$
\mu(\mathscr{P}) \geqslant q\left(q^{2}+q+1\right) .
$$

Наконец, если существует класс $[X]$ со свойством $\mu([X])=0$, то нужное неравенство $\mu(\mathscr{P}) \geqslant s q(q+1)$ получается путем подсчета кратностей точек на прямых, проходящих через $[X]$.

Теперь предположим, что

$$
\mu(\mathscr{P})=s q(q+1)
$$

Тогда по указанным выше причинам существует класс точек $[X]$ такой, что $\mu([X])=0$. Рассмотрим класс $[l]$ соседних линий такой, что в проективной геометрии $\left(\mathscr{P}^{(1)}, \mathscr{L}^{(1)}, J\right)$ выполняется условие $[X] J[l]$, то есть $\bar{X} \subset \bar{l}$. Тогда

$$
\mu([l])=s q .
$$


Рассмотрим проективную плоскость $\left(\mathfrak{P}, \mathfrak{L},\left.\mathfrak{I}\right|_{\mathfrak{P} \times \mathfrak{L}}\right)$, соответствующую прямой $l$ (см. теорему 3) и определим новое мультимножество

$$
\tilde{\mu}: \begin{cases}\mathfrak{P} & \rightarrow \mathbf{N}_{0} \\ {[P] \cap l^{\prime}} & \rightarrow \mu\left([P] \cap l^{\prime}\right), \text { где } \pi\left(l^{\prime}\right)=[l] \\ P_{\infty} & \rightarrow s .\end{cases}
$$

Тогда $\tilde{\mu}$ есть $(s(q+1), s)$-блочное мультимножество и по теореме 5 существуют прямые $\mathfrak{l}_{1}, \ldots, \mathfrak{l}_{s}$ такие, что $\tilde{\mu}(\mathfrak{p})$ есть число прямых $\mathfrak{l}_{j}$ инцидентных точке $\mathfrak{p}$. Все прямые $\mathfrak{l}_{j}$ должны быть инцидентны точке $P_{\infty}$, поэтому $\mu([Y])$ делится на $q$ для всех $[Y] \in \mathscr{P}^{(1)}$.

Определим мультимножество $\eta$ условием

$$
\eta:\left\{\begin{array}{lll}
\mathscr{P}(1) & \rightarrow \mathbf{N}_{0} \\
{[X]} & \rightarrow \mu([X]) / q .
\end{array}\right.
$$

Тогда для класса $[l]$ соседних линий согласно пунктам (d) и (b) теоремы 1

$$
\begin{aligned}
\eta([l]) & =\frac{1}{q} \sum_{[X] J[l]} \mu([X]) \\
& =\frac{1}{q^{2}} \sum_{[X] J[l]} \sum_{l^{\prime} \in[l]} \mu\left([X] \cap l^{\prime}\right) \\
& =\frac{1}{q^{2}} \sum_{l^{\prime} \in[l]} \mu\left(l^{\prime}\right) \geqslant s .
\end{aligned}
$$

Так как $\eta\left(\mathscr{P}^{(1)}\right)=s(q+1)$, мы можем опять использовать теорему 5 для завершения доказательства.

Следствие 2. В любой геометрии $\mathrm{PHG}\left(R_{R}^{3}\right)$, где $R$ - цепное колъцо порлдка $q^{2} c$ полем вычетов порядка $q$, верны равенства

$$
m_{q^{2}+s}\left(R_{R}^{3}\right)=q^{4}+q^{2} s+q s, \quad 0 \leqslant s \leqslant q-1 .
$$

Теорема 7. Справедлива оченка

$$
m_{2}\left(R_{R}^{3}\right) \leqslant \begin{cases}q^{2}+q+1, & \text { если } q \text { четно, } \\ q^{2}, & \text { если } q \text { нечетно. }\end{cases}
$$

Доказательство. Пусть $\mu-$ проективная $(k, 2)$-дуга в $(\mathscr{P}, \mathscr{L}, I)=\operatorname{PHG}\left(R_{R}^{3}\right)$. Для фиксированной прямой $l \in \mathscr{L}$ рассмотрим проективную геометрию $(\mathfrak{P}, \mathfrak{L}, \mathfrak{I} \mid \mathfrak{P} \times \mathfrak{L})$ (см. теорему 3 ). Определим дугу $\tilde{\mu}$ (не обязательно проективную) условиями

$$
\tilde{\mu}: \begin{cases}\mathfrak{P} & \rightarrow \mathrm{N}_{0}, \\ {[X] \cap l} & \rightarrow \mu([X] \cap l), \\ P_{\infty} & \rightarrow 1, \text { если } \mu([X]) \in\{0,1\} \forall[X] J[l], \\ P_{\infty} \rightarrow 0 \text { в противном случае. }\end{cases}
$$

Если $\tilde{\mu}-$ проективное мультимножество, то это - $(K, 2)$-дуга в $\mathrm{PG}(2, q)$, и потому $K \leqslant q+2$, если $q$ четно, и $K \leqslant q+1$, если $q$ нечетно. Если $\tilde{\mu}$ не проективно, то $K=\tilde{\mu}([X])=2$ для некоторого $[X] J[l]$. 
По следствию $1 k \leqslant q^{2}+q+1$ для любого $q$. Будем считать далее, что $q$ нечетно. Если существует класс $[X]$ такой, что $\mu([X])>1$, то $\mu([X])=2$ по теореме 4 . В таком случае для $q$ прямых $[l]$, проходящих через $[X]$ мультимножество $\tilde{\mu}$ проективно в соответствующей $l$ проективной геометрии $\left(\mathfrak{P}, \mathfrak{L},\left.\mathfrak{I}\right|_{\mathfrak{P} \times \mathfrak{L}}\right)$, а для одной прямой $\tilde{\mu}$ не проективно. Отсюда следует нужное неравенство

$$
\mu(\mathscr{P}) \leqslant 2+q(q-1)+0=q^{2}-q+2 .
$$

Если $q$ нечетно и $\mu([X]) \leqslant 1$ для всех $[X]$, то для фиксированного класса $[P]$ со свойством $\mu([P])=1$ получаем, что

$$
\mu(\mathscr{P})=\sum_{[l]:[P] J[l]} \sum_{[X] J[l]} \mu([X])-q \mu([P]) \leqslant q(q+1)-q=q^{2} .
$$

Следствие 3. Если $|R|=q^{2}$ четно, то любая $\left(q^{2}+q+1,2\right)$-дуга $\mu$ в $\mathrm{PHG}\left(R_{R}^{3}\right)$ удовлетворяет условию $\mu([X])=1$ для всех $[X] \in \mathscr{P}^{(1)}$.

Eсли $|R|=q^{2}$ нечетно, то любал $\left(q^{2}, 2\right)$-дуга в $\mathrm{PHG}\left(R_{R}^{3}\right)$ удовлетворяет условию $\mu([X]) \leqslant 1$ для всех $[X] \in \mathscr{P}^{(1)}$, причем все классы $[X]$ кратности $\mu([X])=0$ коллинеарны в $\left(\mathscr{P}^{(1)}, \mathscr{L}^{(1)}, J\right)$.

Доказательство. Приведем доказательство второго утверждения. Мультимножество $\widehat{\mu}: \mathscr{P}^{(1)} \rightarrow \mathbf{N}_{0}$, определяемое равенством $\widehat{\mu}=1-\mu^{(1)}$, есть блочное множество, удовлетворяющее условию $\widehat{\mu}\left(\mathscr{P}^{(1)}\right)=q+1$, и следовательно, это прямая.

В приводимых ниже примерах подразумевается, что все дуги $\mu$ проективны. Это позволяет определять их просто описанием множества Supp $\mu$.

Пример 2. В $\mathrm{PHG}\left(Z_{4}^{3}\right)$ существует $(7,2)$-дуга, состоящая, например, из точек

$$
(1,0,0), \quad(0,1,0), \quad(0,0,1), \quad(1,1,1), \quad(1,3,2), \quad(2,1,3), \quad(3,2,1) .
$$

Однако легко проверить, что такой дуги нет в проективной ельмслевовой плоскости над $F_{2}[x] /\left(x^{2}\right)$. Действительно, в случае существования такой дуги, не нарушая общности рассуждений, можно считать, что она содержит точки

$$
(1,0,0), \quad(0,1,0), \quad(0,0,1), \quad(1,1,1) .
$$

Существует еще ровно три точки, каждая из которых не коллинеарна с двумя из указанных четырех точек:

$$
(1, x+1, x), \quad(x, 1, x+1), \quad(x+1, x, 1) .
$$

Эти три точки коллинеарны. Следовательно, в $\mathrm{PHG}\left(\left(F_{2}[x] /\left(x^{2}\right)\right)^{3}\right)$ существует $(6,2)$ дуга, но не существует $(7,2)$-дуги.

Пример 3. Рассмотрим $(7,2)$-дугу в $\operatorname{PHG}\left(Z_{4}^{3}\right)$. Добавим к этой дуге три точки из некоторой внешней прямой. Полученное множество есть $(10,3)$-дуга в $\operatorname{PHG}\left(Z_{4}^{3}\right)$. В проективной ельмслевовой плоскости над $F_{2}[x] /\left(x^{2}\right)$ также существует $(10,3)$-дуга, например,

$$
\begin{array}{llllll}
(1,1,0), \quad(1,1, x), \quad(1,0,1), \quad(1, x, 1), & (0,1,1), & (x, 1,1), & \\
& (1, x, 0), & (0,1, x), & (x, 0,1), & (1,1,1) .
\end{array}
$$


В остальных примерах мы полагаем $q=3$.

Пример 4. Проективные $(9,2)$-дуги есть в $\operatorname{PHG}\left(Z_{9}^{3}\right)$ и $\operatorname{PHG}\left(\left(F_{3}[x] /\left(x^{2}\right)\right)^{3}\right)$. Таковы, например, множества

$$
(1,0,0), \quad(0,1,0), \quad(0,0,1), \quad(1,1,3), \quad(1,3,1), \quad(3,1,1), \quad(1,2,5),
$$

$$
(1,4,2), \quad(1,8,7)
$$

в $\operatorname{PHG}\left(2, Z_{9}\right)$ или

$$
\begin{array}{rrrr}
(1,0,0), \quad(0,1,0), \quad(0,0,1), \quad(1,1,1), & (1, x, 2+2 x), \\
(1,1+x, 2 x), & (1,1+x, 2+x), \quad(1,2,2+x), \quad(x, 1,2),
\end{array}
$$

в $\operatorname{PHG}\left(2, F_{3}[x] /\left(x^{2}\right)\right)$.

Пример 5. Рассмотрим овал (множество из $q+1$ точек в $\mathrm{PG}(2, q)$, не содержащее трех коллинеарных точек)

$$
\mathscr{O}=\left\{\left[P_{0}\right], \ldots,\left[P_{3}\right]\right\}
$$

в $\pi(\Pi) \cong \mathrm{PG}(2,3)$. В каждом классе $\left[P_{i}\right]$ выберем три коллинеарных точки $X_{i, 1}$, $X_{i, 2}, X_{i, 3}$ из ельмслевовой геометрии так, что для любой прямой $l$, содержащей эти точки, ее образ $\pi(l)$ есть касательная к $\mathscr{O}$ в точке $\left[P_{i}\right]$. Обозначим через $\left[A_{0}\right],\left[A_{1}\right]$, $\left[A_{2}\right]$ внутренние точки (точки, не принадлежащие касательным) овала $\mathscr{O}$. Выберем две точки в каждом из классов соседних точек $\left[A_{i}\right]$, скажем $A_{i, 1}, A_{i, 2} \in\left[A_{i}\right]$, таким образом, что для любой прямой $l$, содержащей $A_{i, 1}, A_{i, 2}$, выполняется равенство

$$
\pi(l)=\left\langle\left[A_{i}\right],\left[A_{i+1}\right]\right\rangle
$$

(индексы выбираются по модулю 3). Нетрудно проверить, что каждая прямая в $\operatorname{PHG}\left(R_{R}^{3}\right)$ инцидентна не более трем точкам из множества $\left\{X_{i, j}, A_{u, v}\right\}$. Так как мы не использовали конкретного строения цепного кольца $R$, определенное таким образом множество из 18 точек образует $(18,3)$-дугу одновременно как в $\operatorname{PHG}\left(Z_{9}^{3}\right)$, так и в $\operatorname{PHG}\left(\left(F_{3}[x] /\left(x^{2}\right)\right)^{3}\right)$.

Пример 6. Выберем точки $X_{i, j}$ так же, как и в примере 5. Затем выберем четыре точки, образующие овал в каждом из классов $\left[A_{i}\right]$ (см. теорему 2). Полученное множество из 24 элементов есть $(24,4)$-дуга.

Пример 7. Выберем точки $X_{i, j}$ так же, как и в примере 5 . Выберем в каждом классе $\left[A_{i}\right]$ шесть точек так, что они лежат на двух параллельных прямых, имеющих направление $\left\langle\left[A_{i}\right],\left[A_{i+1}\right]\right\rangle$. Полученное таким образом множество из 30 элементов есть $(30,5)$-дуга. Оказывается, эта дуга неполна. Присоединение к ней произвольной точки из любого класса $\left[P_{i}\right]$ дает $(31,5)$-дугу.

Пример 8. Рассмотрим те же самые классы $\left[P_{i}\right],\left[A_{j}\right]$, что и в примере 5 . В каждом из них выберем шесть точек так, что они расположены на двух параллельных прямых. Направления этих параллельных прямых выбираются опять, как в примере 5. Полученное множество есть $(42,6)$-дуга.

Пример 9. В каждом из классов $\left[P_{i}\right]$ выберем шесть точек, содержащихся в двух параллельных прямых, имеющих направление касательной к $\mathscr{O}$ в этих точках. В каждом из классов $\left[A_{0}\right],\left[A_{1}\right],\left[A_{2}\right]$ выберем все 9 точек. Полученное множество есть $(51,7)$-дуга. 
Пример 10. Рассмотрим ту же самую ельмслевову геометрию, что и в предыдущих примерах. Выберем три прямые линии $l_{1}, l_{2}, l_{3}$, не имеющие общей точки, и такие, что $l_{1} \not \Varangle l_{2} \not \propto l_{3} \not \Varangle l_{1}$. Определим мультимножество $\mu$ условием

$$
\mu(P)= \begin{cases}1, & \text { если } P \not l_{1}, P \notin l_{2}, P \notin l_{3}, \\ 0, & \text { в противном случае. }\end{cases}
$$

Это мультимножество есть $(64,8)$-дуга. В общем случае для любой геометрии $\operatorname{PHG}\left(R_{R}^{3}\right)$, где $|R|=q^{2}, R / \operatorname{rad} R \cong F_{q}$, мы можем построить таким образом $\left(q^{4}-2 q^{2}+1, q^{2}-1\right)$-дугу.

Известные значения и оценки параметра $m_{n}\left(R_{R}^{3}\right)$ для ельмслевовых плоскостей над цепными кольцами из 4 и 9 элементов приведены в таблице в конце статьи. Некоторые из приведенных верхних границ отличаются от значений, указанных в следствии 1. Следующие три замечания поясняют эти уточнения.

Замечание 1. Если $|R|=4$, то в $\operatorname{PHG}\left(R_{R}^{3}\right)$ нет проективной $(11,3)$-дуги. Допустим, что $\mu: \mathscr{P} \rightarrow \mathbf{N}_{0}$ есть $(11,3)$-дуга. По теореме 4 для любого $[X] \in \mathscr{P}^{(1)}$ справедливо неравенство $\mu([X]) \leqslant 2$. Допустим, что существуют четыре класса соседних точек, скажем $\left[X_{1}\right], \ldots,\left[X_{4}\right]$, которые образуют овал в $\pi(\Pi)$ и таких, что $\mu\left(\left[X_{i}\right]\right)=2$. Пусть

$$
\left\{A_{1}, A_{2}\right\}=\left[X_{1}\right] \cap \operatorname{Supp} \mu \text {. }
$$

Тогда для любой прямой $s$, содержащей точки $A_{1}, A_{2}$, ее образ $\pi(s)$ содержит ровно одну из точек $\left[X_{2}\right],\left[X_{3}\right],\left[X_{4}\right]$, скажем $\left[X_{2}\right]$. Пусть

$$
\left\{B_{1}, B_{2}\right\}=\left[X_{2}\right] \cap \operatorname{Supp} \mu .
$$

Аналогично, для любой прямой $t$, удовлетворяющей условию $t \supset\left\{B_{1}, B_{2}\right\}$, прямая $\pi(t)$ содержит точно одну из точек $\left[X_{3}\right],\left[X_{4}\right]$, скажем $\left[X_{3}\right]$. Если $\left[Y_{1}\right]-$ третья точка на прямой $\left\langle\left[X_{1}\right],\left[X_{2}\right]\right\rangle$ и $\left[Y_{2}\right]$ - третья точка на прямой $\left\langle\left[X_{2}\right],\left[X_{3}\right]\right\rangle$, то $\mu\left(\left[Y_{1}\right]\right)=$ $\mu\left(\left[Y_{2}\right]\right)=0$. Следовательно, $\mu(\mathscr{P}) \leqslant 5 \cdot 2=10$, что противоречит предположению.

Обозначим через $f_{i}$ число классов $[X]$ соседних точек со свойством $\mu([X])=i$. Приведенные выше рассуждения показывают, что $f_{2}<5$, следовательно, $f_{1}=3$, $f_{2}=4$ и $f_{i}=0$ для $i \neq 1,2$. Более того, среди классов соседних точек $\left[X_{i}\right]$ со свойством $\mu\left(\left[X_{i}\right]\right)=2$ три класса, скажем $\left[X_{1}\right],\left[X_{2}\right],\left[X_{3}\right]$, коллинеарны. Пусть

$$
\left\{A_{1}, A_{2}\right\}=\left[X_{4}\right] \cap \operatorname{Supp} \mu
$$

и пусть $s$ - прямая такая, что $s \supset\left\{A_{1}, A_{2}\right\}$ и $\pi(s)$ инцидентна $\left[X_{1}\right]$. Как и прежде, можно утверждать, что три класса $[Y]$, лежащих на прямой, проходящей через точки $\left[X_{1}\right]$ и $\left[X_{4}\right]$ не содержат точек из $\operatorname{Supp} \mu$. Это невозможно, так как $f_{0}=0$.

Замечание 2. Если $|R|=9$, то в $\operatorname{PHG}\left(R_{R}^{3}\right)$ не существует проективной $(20,3)$-дуги. Пусть $\mu$ есть $(20,3)$-дуга. Тогда по теореме 4 для любого класса $[X]$ верно неравенство $\mu([X]) \leqslant 3$. Допустим, что $[X]-$ класс соседних точек такой, что $\mu([X])=3$, и пусть

$$
[X] \cap \operatorname{Supp} \mu=\left\{X_{1}, X_{2}, X_{3}\right\} .
$$

Нетрудно видеть, что точки $X_{1}, X_{2}, X_{3}$ коллинеарны. Тогда для любой прямой $s$, содержащей эти точки, любая точка $[Y]$, принадлежащая прямой $[s]$ в геометрии $\pi(\Pi)$, 
удовлетворяет условию $\mu([Y])=0$. Допустим, далее, что $[t]-$ прямая, содержащая точку $[X]$ в $\pi(\Pi)$, и такая, что

$$
\sum_{[Y] J[t]} \mu([Y])=9
$$

Хорошо известно, что проективная $(9,3)$-дуга в $\mathrm{PG}(2,3)$ состоит из всех точек плоскости без некоторой прямой. Отсюда по теореме 3 получаем, что существует класс соседних точек $\left[Y_{0}\right]$, инцидентный $[t]$, с условием $\mu\left(\left[Y_{0}\right]\right)=0$. Следовательно, для двух других точек $\left[Y_{1}\right],\left[Y_{2}\right]$ из $[t]$

$$
\mu\left(\left[Y_{1}\right]\right)=\mu\left(\left[Y_{2}\right]\right)=3 .
$$

Таким образом, существует по крайней мере 7 точек $[Z]$ со свойством $\mu([Z])=0$ и потому $\mu(\mathscr{P}) \leqslant 6 \cdot 3=18$, что противоречит предположению.

Пусть теперь $\mu([Y]) \leqslant 2$ для всех $[Y]$, и пусть параметры $f_{i}$ определены, как и в предыдущем замечании. Допустим, что $[X]-$ класс со свойством $\mu([X])=2$ и

$$
\left\{A_{1}, A_{2}\right\}=[X] \cap \operatorname{Supp} \mu \text {. }
$$

Тогда любая прямая $\pi(s)$ такая, что $s \supset\left\{A_{1}, A_{2}\right\}$, инцидентна не более чем двум классам, содержащим по две точки из Supp $\mu$. Подсчитывая число пар $([Y],[t])$ таких, что $[X] J[t]$ и $\mu([Y])=2$, мы получаем, что

$$
2 f_{2}+4\left(13-f_{2}\right) \geqslant 4 f_{2}
$$

следовательно, $f_{2} \leqslant 8$.

Допустим, что не существует пустых классов, то есть таких, что $\mu([X])=0$, и существует четыре класса, содержащих по две точки из $\operatorname{Supp} \mu$, скажем $[X],[Y],[Z]$, $[W]$, образующих овал $\mathscr{O}$ в $\pi(\Pi)$. Если

$$
\left\{X_{1}, X_{2}\right\}=[X] \cap \operatorname{Supp} \mu
$$

и $s-$ прямая со свойством $s \supset\left\{X_{1}, X_{2}\right\}$, то $\pi(s)$ - касательная к $\mathscr{O}$. Другие классы, содержацие по две точки из $\operatorname{Supp} \mu$, могут быть только внешними точками к $\mathscr{O}$ в $\left(\mathscr{P}^{(1)}, \mathscr{L}^{(1)}, J\right)$. Заметим также, что мы не можем иметь 2 точки из носителя во всех внешних точках. Следовательно, если $f_{0}=0$, то $f_{2} \leqslant 6$.

Объединяя эти результаты, мы получаем, что если существует $(20,3)$-дуга, то $f_{0}=1, f_{1}=4, f_{2}=8$. Допустим, что $\mu$ - такая дуга. Определим дугу $\eta$ в $\left(\mathscr{P}^{(1)}, \mathscr{L}^{(1)}, J\right)$ условиями $\eta([X])=1$, если $\mu([X])=2$ и $\eta([X])=0$ в противном случае. Пусть $a_{i}$ есть число линий таких, что $\eta(l)=i$. Тогда должны выполняться соотношения

$$
\begin{aligned}
a_{0}+a_{1}+a_{2}+a_{3}+a_{4} & =13 \\
a_{1}+2 a_{2}+3 a_{3}+4 a_{4} & =32 \\
a_{2}+3 a_{3}+6 a_{4} & =28
\end{aligned}
$$


Таблица 1. Точные значения и оценки параметра $m_{n}\left(R_{R}^{3}\right)$ для $|R| \in\{4,9\}$

\begin{tabular}{|r|r|r|r|r|}
\hline & \multicolumn{4}{|c|}{$R$} \\
\cline { 2 - 5 }$n$ & $Z_{4}$ & $F_{2}[x] /\left(x^{2}\right)$ & \multicolumn{1}{|c|}{$Z_{9}$} & $F_{3}[x] /\left(x^{2}\right)$ \\
\hline 2 & 7 & 6 & 9 & 9 \\
3 & 10 & 10 & $18-19$ & $18-19$ \\
4 & 16 & 16 & $24-33$ & $24-33$ \\
5 & 22 & 22 & $31-40$ & $31-40$ \\
6 & 28 & 28 & $42-52$ & $42-52$ \\
7 & & & $51-62$ & $51-62$ \\
8 & & & $64-75$ & $64-75$ \\
9 & & & 81 & 81 \\
10 & & & 93 & 93 \\
11 & & & 105 & 105 \\
12 & & & 117 & 117 \\
\hline
\end{tabular}

Более того, $a_{1}+a_{2} \geqslant 8$, поскольку для класса $[X]$, удовлетворяющего условию

$$
[X] \cap \operatorname{Supp} \mu=\left\{A_{1}, A_{2}\right\}
$$

прямая $\left\langle A_{1}, A_{2}\right\rangle$ проходит еще не более чем через один класс с двумя точками из Supp $\mu$. Отсюда следует, что возможен лишь один набор значений $a_{i}$

$$
a_{0}=0, \quad a_{1}=1, \quad a_{2}=7, \quad a_{3}=3, \quad a_{4}=2 .
$$

Теперь легко проверить, что все, за исключением одного из классов $[X]$, удовлетворяющих условию $\mu([X])=2$, обладают тем свойством, что прямые, проходящие через точки $A_{1}, A_{2} \in[X] \cap \operatorname{Supp} \mu$, проходят через единственный класс $[Y]$, для которого $\mu([Y])=0$. Отсюда следует, что в рассматриваемой ельмслевовой плоскости существуют 4 коллинеарных точки, что невозможно для $(20,3)$-дуги.

Замечание 3. В случае $|R|=9$ верхние границы для $m_{n}\left(R^{3}\right), n=5,6,7,8$, приводимые ниже в таблице, лучше, чем границы, вытекающие из следствия 1. Например, если $n=6$, то следствие 1 дает неравенство $m_{6}\left(R^{3}\right) \leqslant 53$, и позволяет также утверждать, что если $(53,6)$-дуга существует, то существует класс $[X]$ соседних точек, содержащий точно 5 точек из носителя. Так как максимальный размер овала в $\mathrm{PG}(2,3)$ равен 4 , существует прямая, которая пересекается с $[X]$ в трех точках. Теперь мы можем повторить доказательство пункта (b) теоремы 4 для доказательства несуществования $(53,6)$-дуги. Остальные случаи рассматриваются аналогично.

Исследования второго автора были выполнены при поддержке стипендией Александра фон Гумбольдта. Оба автора благодарны Вернеру Хайзе за многочисленные советы и предложения в процессе написания этой статьи. Они признательны А. А. Нечаеву за критические замечания и полезные комментарии, способствовавшие улучшению статьи. 


\section{Список литературы}

1. Нечаев А. А., Линейные коды и полилинейные рекурренты над конечными кольцами и квазифробениусовыми модулями. Докл. АН РФ (1995) 345, №4, 451-454.

2. Нечаев А. А., Кузьмин А. С., Марков В. Т., Линейные коды над конечными кольцами и модулями. Фундаментальная и прикладная математика (1996) 2, №3, 195-254.

3. Nechaev A. A., Linear codes over modules and over spaces. MacWilliams' identity. Proc. 1996 IEEE Int. Symp. Inf. Theory and Appl., Victoria, Canada, 1996, 35-38.

4. Nechaev A. A., Kuzmin A. S., Linearly presentable codes. Proc. 1996 IEEE Int. Symp. Inf. Theory and Appl., Victoria, Canada, 1996, 31-34.

5. Nechaev A. A., Kuzmin A. S., Trace function on a Galois ring in coding theory. Lect. Notes Comp. Sci. (1997) 1255, 277-290.

6. Nechaev A. A., Kuzmin A. S., Formal duality of linear presentable codes over a Galois field. Lect. Notes Comp. Sci. (1997) 1255, 263-276.

7. Нечаев А. А., Хонольд Т., Полновесные модули и представления кодов. Пробл. передачи. информ. (1999) 35, №3, 18-39.

8. Greferath M., Schmidt S. E., Gray isometries for finite chain rings. IEEE Trans. Inform. Theory (1999) 45, 2522-2524.

9. Kurakin V. L., Kuzmin A. S., Markov V. T., Mikhalev A. V., Nechaev A. A., Linear codes and polylinear recurrences over finite rings and modules (a survey). Lect. Notes Comp. Sci. 1719, 365-391.

10. Honold T., Landjev I., Linearly representable codes over chain rings. Abhandlungen aus dem math. Seminar der Univ. Hamburg (1999) 69, 187-203.

11. Honold T., Landjev I. Linear codes over finite chain rings. Electronic J. Combinatorics (2000) 7 №11.

12. Нечаев А. А., Конечные кольца главных идеалов. Матем. сб. (1973) 91, №3, 350-366.

13. Clark W. E., Drake D. A., Finite chain rings. Abhandlungen aus dem math. Seminar der Univ. Hamburg (1974) 39, 147-153.

14. McDonald B. R., Finite rings with identity. Marcel Dekker, New York, 1974.

15. Нечаев А. А., Код Кердока в циклической форме. Дискретная математика (1989) 1, №4, 123-139.

16. Hammons A. R., Kumar P. V., Calderbank A. R., Sloane N. J. A., Solé P., The $Z_{4}$-linearity of Kerdock, Preparata, Goethals, and related codes. IEEE Trans. Inform. Theory (1994) 40, №2, 301-319.

17. Kurakin V. L., Kuzmin A. S., Mikhalev A. V., Nechaev A. A., Linear recurring sequences over rings and modules. J. Math. Sci. (1955) 76, №6, 2793-2915.

18. Hirschfeld J. W. P., Projective geometries over finite fields. Oxford Univ. Press, Oxford, 1998.

19. Hirschfeld J. W. P., Storme L., The packing problem in statistics, coding theory and finite projective spaces. Preprint, 2000.

20. Dembowski P., Finite geometries. Springer, Berlin, 1968.

21. Klingenberg W. Projektive und affine Ebenen mit Nachbarelementen. Math. Z. (1954) 60, 384-406.

22. Kreuzer A., Hjelmslevsche Inzidenzgeometrie. Ein Bericht. Bericht TUM-M9001, Technische Univ. München, Beiträge zur Geometrie und Algebra, 1990, Jan. Nr. 17. 
23. Veldkamp F. D., Geometry over rings. In: Handbook of incidence geometry. Buildings and foundations. Elsevier, Amsterdam, 1995, pp. 1033-1084.

24. Dieudonné J., Remarks on quasi-Frobenius rings. Illinois J. Math. (1958) 1-2, 346-354.

25. Raghavendran R., Finite associative rings. Compositio Mathematica (1969) 21, 195-229.

26. Kleinfeld E., Finite Hjelmslev planes. Illinois J. Math. (1959) 3, 403-407.

27. Artmann B., Hjelmslev-Ebenen mit verfeinerten Nachbarschaftsrelationen. Math. Z. (1969) 112, 163-180.

28. Drake D. A., On n-uniform Hjelmslev planes. J. Comb. Theory (1970) 9, 267-288.

Статья поступила 26.05.1999.

Переработанный вариант поступил 30.12.2000. 